\title{
Pre-Identification of Lactic Acid Bacteria Isolated During Fermentation Process of Egyptian Kishk
}

\author{
Amel Ibrahim, Nabil M. Kamal, Sameh Awed, Aisha El-Attar ${ }^{1}$
}

\begin{abstract}
The cereal-based fermented food Egyptian Kishk constitutes a major source of high quality dietary nutrients for Upper Egyptians and is popular among all social strata. Lactic acid bacteria were one of the main flora which is responsible for fermentation. To-date, kishk is still traditional making and the diversity of LAB in Kishk has not been fully investigated and improving the Kishk throughout using selected cultures for its processing has never been established. In this work, LAB have been isolated from samples collected during traditional making the Kishk. The obtained results showed that the dominant lactic acid bacteria isolates were obligatory heterofermentative and Enterococci spp. The homofermentative LAB were found in the first steps of the fermentation. Homofermentative and facultative heterofermentative of lactobacilli were found in the first fermentation process steps and then- almost disappeared after that in Kishk. On the other hand obligatory hetero fermentative and Enterococci spp. were detected in all steps of kishk manufacturing while Lactococcus was a minor isolate during fermentation process. The majority of isolates are $\gamma$ - haemolytic except one isolate of Enterococci spp. which was $\beta$ haemolytic. There are significant differences $(P \leq$ 0.01) in organoleptic characteristics (colour, taste, smell, texture, appearance) and overall acceptability among all isolates used to ferment the milk. Fifteen isolates that received the highest levels of overall organoleptic properties are selected as starter culture in improving the Kishk making.
\end{abstract} Kishk

Key words: Lactic acid bacteria, Laban Zeer and

\section{INTRODUCTION}

Kishk is typically prepared by mixing Laban Zeer (butter milk separated from freshly drawn milk and left to sour in an unglazed earthenware container: the "Zeer") with coarsely ground parboiled wheat. The locally grown mature freshly harvested whole wheat is boiled in a metal barrel on an open fire until soft then sun dried for 24 hours. The following day, the dried grains are moistened with water (about $20 \%$ ), cracked and husked by winnowing, and then sized as coarse and fine. It is recommended that coarse burghol be used for kishk making. The concentrated Laban Zeer that is fermented for at least 40 days is mixed with the moistened coarsely ground parboiled wheat in a large earthenware magour at ratio of $2: 1$ to $4: 1$, to produce a heavy paste called "Hama" Ahmed and Hassan-Wassef (2010). The milk cereal mixture is then allowed to ferment again for about 24 hours after which it is kneaded with the addition of more of the fermented salted milk diluted with a little water to reach the required consistency. Alternatively, the fermentation of the Hama allowed continuing for a further 24 hours. The resultant mass is thoroughly mixed incorporating the aromatic spices before cutting into unformed chunks (of about $3 \mathrm{~cm}$ in diameter) or shaped into small balls of about $2 \mathrm{~cm}$ in diameter, dried for 7-8 days in the hot shade or sun. (Abd El- Salam, 2011)

The microbiological analysis of Kishk is quite complex. Differences in the traditional methods employed during the manufacture of Kishk can influence the compositional, nutritional properties and microbiological characteristics of the product (Tamime and O'Connor, 1995). Lactic acid bacteria and yeasts constituted the main microbial flora during fermentation process of Egyptian Kishk (Demerdash, 1960). The chemical and microbial changes during preparation of wheat yoghurt, Kishk, have been studied (Demerdash, 1960; Shaker et al., 1980; Hamad and Fields, 1982 ;Damir et al., 1992). From the documented literatures, it seems that very little work has been attempted to identify the fermenting organisms from Laban Zeer to Kishk in this background.

The present study aims at the isolation and preidentification of lactic acid bacteria during actual Kishk manufacturing and studies their hemolytic activity, in addition to screening the isolates for flavor acceptability in fermented milk. The obtained results will be used to select appreciate strains to be as starter culture in producing second generation of kishk.

\section{MATERIALS AND METHODS}

\section{Sample collection:}

Sixty nine Laban Zeer samples, seven samples from each of (dough and Hama) and ten samples of Kishk were collected in sterile plastic bags from Bani -Mazar, Samlot, Abu-korkas, Mallawi and Der- Maus (Cites of El-Minya Governorate, Egypt during July, 2011 and 2012). The samples were transferred in ice box and then stored. Laban Zeer samples were evaluated for flavor and texture acceptability by 22 consumer panelists (all

\footnotetext{
${ }^{1}$ Department of Dairy Science and Technology, Faculty of Agriculture, Shatby, Laboratory of Microbial Biochemistry, Alexandria University Received April 1, 2015, Accepted April 26, 2015
} 
of them were familiar with Laban Zeer). Twenty five samples which accepted in flavor and texture were selected to isolate the lactic acid bacteria. Other seven samples of Laban Zeer, Dough, Hama and Kishk which collected during the actual manufacture process were also used for isolation the lactic acid bacteria.

\section{Isolation of lactic acid bacteria (LAB) strains:}

Three grams of each sample were cultured aseptically into $30 \mathrm{ml}$ of sterilized reconstituted skimmed milk and were incubated until coagulation at $30{ }^{\circ} \mathrm{C} ; 37^{\circ} \mathrm{C}$ and $42{ }^{\circ} \mathrm{C}$. Coagulated samples were then streaked on M17 (Biolife Italy), ST and SF agar media. These samples streaked also on MRS and Rogosa agar (Difco, USA) which were incubated under anaerobic condition using the gas pak system (GENER box anae indicator Biomerieux) at $30{ }^{\circ} \mathrm{C}, 37{ }^{\circ} \mathrm{C}$ and $42{ }^{\circ} \mathrm{C}$ for $48 \mathrm{hr}$. The cultures were streaked on suitable media for their purification. The purified strains were stored at $-20{ }^{\circ} \mathrm{C}$ in sterile reconstituted skim milk $(12.5 \% \mathrm{~W} / \mathrm{V})$ supplemented with $15 \%$ glycerol (El-Soda et al., 2003)

\section{Pre Identification tests:}

The isolates were roughly classified at genus level according to the Criteria of Sharpe (1979) and Axelsson (1993). Tests applied on all isolates were: Gram positive and catalase negative, consequently they were tested for growth at $10^{\circ} \mathrm{C}$ for 10 days, $45^{\circ} \mathrm{C}$ for $48 \mathrm{hr}$, and $\mathrm{CO}_{2}$ production from glucose. For cocci isolates, growth on SF broth medium and in the presence of 6.5 $\% \mathrm{NaCl}$ were also considered.

\section{Hemolytic activity:}

Hemolytic activity was evaluated using blood agar base 2 (Oxoide) plates containing $5 \%(\mathrm{v} / \mathrm{v})$ human blood (EL-Shatby hospital, Alexandria) and incubated at 30,37 and $42{ }^{\circ} \mathrm{C}$ for $48 \mathrm{hr}$. $\alpha$ - and $\beta$ - hemolytic reactions were recorded by observation of greenish colonies and a clear zone around the colonies respectively. The assay was performed in duplicate. Streptococcus pyogenes MGAS 15252 serotype M 59 (obtained from department of Food Technology, Arid Land Cultivation and Development Institute, City of Scientific Research and Technology Applications) was used as $\beta$-hemolytic control strain (Maragkoudakis et al., 2006).

\section{Flavor potential for products having specific flavours:}

Fermented milk prepared by adding 3\% of active single tested strain at optimum temperature until milk coagulated at $\mathrm{pH}$ 4.6. The fermented milk was kept at 4 ${ }^{\circ} \mathrm{C}$ for $18 \mathrm{~h}$. Final products were evaluated for organoleptic characteristics (color, taste ,smell, texture ,appearance) and overall acceptability by 15 panelists included post graduate- students and staff members at
Faculty of Agriculture, Alexandria university; using ten point hedonic scale ranging from excellent $($ score $=10$ ) to very poor $($ score $=0$ ) as extremes. Also the panelists were asked to list any flavour defects (Abdel Rahman et al., 2009).

\section{Statistical analysis:}

The experimental design was a completely randomized design with 15 replications in the experiment. F-test, and analysis of variance of treatments difference was performed according to (Steel and Torrie, 1984). Statistical analysis was done by, ANOVA, F-test, and L.S.D procedures available within the SAS software package (version 9.13 2008).

\section{RESULTS AND DISCUSSION}

\section{Pre-identification tests of isolated lactic acid bacteria:}

Pre-identification results of obtained isolates during Egyptian Kishk manufacturing are illustrated in Table (1). Three hundred and eight isolates were phenotypically identified, all of the isolates were Gram positive and catalase negative. The results showed that Enterococcus spp. isolates were found in high proportion $(94.1,93.7,94$ and 95.8\%) for cocci in Laban Zeer, Dough, Hama and Kishk respectively. The highly number of Enterococcus spp. was in agreement with the results that obtained by El Soda et al.(2003). They found a relatively high number of Enterococcus spp in Egyptian dairy products (Ras, Domiatti and Kareish cheese, mish, cream, butter, Zabady and Laban Rayeb). Lactococcus spp. was detected in minor proportion (5.8, 6.3, 6 and $4.2 \%$ ) in Laban Zeer, Dough, Hama and Kishk respectively for cocci. As likewise reported by Abd El-Malek and Demerdash (1970), the presence of Lactococcus lactis and Lactococcus cremoris in Laban Zeer.

Lactobacillus isolates were classified into 3 groups (Kandler and Weiss, 1986). The domination of obligatory heterofermentative lactobacilli isolates (group C) $(56.9,54.8,70.9$ and $79.1 \%$ ) were observed in samples followed by facultative heterofermentative lactobacilli isolates (group B) $(23.8,38.7,25.8$ and 16.6 $\%$, followed by obligatory homofermentative lactobacilli isolates (group A) (19.2, 6.5, 3.3 and 8.3\%) in Laban Zeer, Dough, Hama and Kishk respectively for lactobacilli.

This study presented the domination of heterofermentative isolates while Demerash (1960) has reported the homofermentative LAB were constituted the main microbial flora (about $84 \%$ of the total microflora) during the fermentation process of Egyptian Kishk. This diversity of lactic acid bacteria is relative and depends primarily on the source of fermented 
buttermilk which was used in Kishk manufacturing kind (Abou-Donia, 1984).

\section{Following Lactic acid bacteria during Egyptian kishk manufacturing steps:}

Table (2) shows the isolated lactic acid bacteria from collected samples during the actual Kishk manufacture steps (Laban Zeer, Dough, Hama and Kishk). The results in (Table 2 and Figure 1) shows that Enterococcus was found in all steps of all samples during actual manufacturing. This result was different in what was reported by Atia and Khattab (1985) who detected Enterococcus faecium in only one out of eight samples of tested Kishk, this difference might be attributed to the kind of sour milk used in Kishk preparation as well as the temperature and time of fermentation and drying. The chemical compositions have also a role on the microbial diversity of tested samples. On the other hand Lactococcus was found in one sample (sample 11) during the first steps of manufacture (Laban Zeer, Dough and Hama). The presence of this organism in the first steps due to the low salt content $(3.98,3.68$ and $1.98 \%$ ) respectively compared with the last step (Kishk $4.74 \%$ ). Generally, group $\mathrm{C}$ was the dominant isolates in all steps of all samples except (sample 64), as it was found in Laban Zeer and then was not detected after that. This result referred to the low numbers of group $\mathrm{C}$ at Laban Zeer samples when compared to Dough,Hama and kishk samples. In all samples, almost group B was found in Laban Zeer and Dough, sometimes found in Hama. Group A was a minor isolates which was isolated from only one sample (sample 63) at the first steps. Similar results were reported by El-Sadek et al., (1958).

\section{Hemolytic activity:}

Hemolytic activity is a trait associated with virulence in some food-associated microorganisms, such as enterococci (Jett et al., 1994 and Franz et al., 1999). In this study, all isolates referring to Enterococcus did not have any hemolytic activity ( $\gamma$-hemolytic) except only one strain (125RZ) that was $\beta$ - hemolytic (Figure 2). While no hemolytic activity was observed for the five isolates of Lactococcus. These results are in agreement with (Maragkoudakis et al., 2009) who reported that there is no hemolytic activity for lactococci of dairy origin. None of the examined Lactobacillus isolates exhibited $\beta$-hemolytic activity when grown in human blood agar base 2. Most of lactobacilli isolates (214 isolates) were $\gamma$-hemolytic (i.e. no hemolytic), while two isolates exhibited $\alpha$-hemolytic (85RZ and 59RZ). These results agreed with those reported by Maragkoudakis et al. (2006).

\section{Off-flavour test:}

The isolates which had $\gamma$-hemolytic activity were evaluated for organoleptic characteristics (color, taste, smell, texture, appearance) and overall acceptability by panelists. The results showed that there were significant differences $(\mathrm{P} \leq 0.01)$ among all isolates in color, taste, smell, texture, appearance and overall (Table 3). The percentage of selected samples (using different isolates) according to the overall organoleptic characteristics scores were done using the ratio between the accepted overall (overall code 2) to all tested isolates. The overall acceptability for tested isolates were (16.66, 13.20 and $0.75 \%$ ) of group A, group B and group C respectively. While, the overall acceptability for tested isolates of Enterococci and Lactococci were (1.17\% and $20 \%$ ) respectively.

Table 1. Pre-identification of isolated LAB during Egyptian Kishk manufacturing

\begin{tabular}{|c|c|c|c|c|c|}
\hline \multirow[b]{2}{*}{ Genus } & \multicolumn{4}{|c|}{ Source } & \multirow[b]{2}{*}{ Total $^{\mathrm{a}}$} \\
\hline & $\begin{array}{l}\text { Laban Zeer } \\
\text { (32samples) }\end{array}$ & $\begin{array}{c}\text { Dough } \\
\text { (7 samples) }\end{array}$ & $\begin{array}{c}\text { Hama } \\
\text { (7 samples) }\end{array}$ & $\begin{array}{c}\text { Kishk } \\
\text { (10samples) }\end{array}$ & \\
\hline Enterococcus spp. & $32(94.1 \%)$ & $15(93.7 \%)$ & $16(94 \%)$ & $23(95.8 \%)$ & $86(94.5 \%)$ \\
\hline Lactococcus spp. & $2(5.8 \%)$ & $1(6.3 \%)$ & $1(6 \%)$ & $1(4.2 \%)$ & $5(5.5 \%)$ \\
\hline Total cocci & 34 & 16 & 17 & 24 & 91 \\
\hline $\begin{array}{l}\text { homofermentative lactobacill } \\
\text { (group A) }\end{array}$ & $25(19.2 \%)$ & $2(6.5 \%)$ & $1(3.3 \%)$ & $2(8.3 \%)$ & $30(13.8 \%)$ \\
\hline $\begin{array}{l}\text { Facultative heterofermentative } \\
\text { lactobacilli(group B) }\end{array}$ & $31(23.8 \%)$ & $12(38.7 \%)$ & $8(25.8 \%)$ & $4(16.6 \%)$ & $55(25.34 \%)$ \\
\hline $\begin{array}{l}\text { obligatory heterofermentative } \\
\text { lactobacilli(group C) }\end{array}$ & $74(56.9 \%)$ & $17(54.8 \%)$ & $22(70.9 \%)$ & $19(79.1 \%)$ & $132(60.8 \%)$ \\
\hline Total lactobacilli & 130 & 31 & 31 & 25 & 217 \\
\hline Total $^{\mathrm{b}}$ & 164 & 47 & 48 & 49 & 308 \\
\hline
\end{tabular}


Table 2. Pre- identification tests of Lactic acid bacteria isolates

\begin{tabular}{|c|c|c|c|c|c|c|c|c|}
\hline \multirow[b]{2}{*}{ Samples } & \multirow[b]{2}{*}{ Isolates } & \multicolumn{6}{|c|}{ Pre - identification test } & \multirow[b]{2}{*}{ Pre- identification } \\
\hline & & Catalase & $\begin{array}{c}\text { Growth } \\
\text { at } \\
10^{\circ} \mathrm{C} \\
\end{array}$ & $\begin{array}{c}\text { Growth } \\
\text { at } \\
45^{\circ} \mathrm{C} \\
\end{array}$ & $\begin{array}{c}\mathrm{CO}_{2} \\
\text { production }\end{array}$ & $\begin{array}{c}\text { SF } \\
\text { growth }\end{array}$ & $\begin{array}{c}\text { Growth } \\
\text { at } 6.5 \% \\
\mathrm{NaCl} \\
\end{array}$ & \\
\hline \multicolumn{9}{|c|}{ Sample 6} \\
\hline \multirow{5}{*}{$6 \mathrm{~L}$} & $44 \mathrm{RZ}$ & - & + & + & - & + & + & Enterococcus spp. \\
\hline & $45 \mathrm{RZ}$ & - & + & + & - & + & + & Enterococcus spp. \\
\hline & $46 \mathrm{RZ}$ & - & + & - & - & & & G.B \\
\hline & $47 \mathrm{RZ}$ & - & - & + & + & & & G.C \\
\hline & 48RZ & - & - & + & + & & & G.C \\
\hline \multirow{6}{*}{$6 \mathrm{D}$} & 58RZ & - & + & + & - & + & + & Enterococcus spp. \\
\hline & 59RZ & - & + & - & - & & & G.B \\
\hline & $60 \mathrm{RZ}$ & - & + & + & - & & & G.B \\
\hline & 61RZ & - & - & + & + & & & G.C \\
\hline & $62 \mathrm{RZ}$ & - & - & + & + & & & G.C \\
\hline & $63 \mathrm{RZ}$ & - & - & + & + & & & G.C \\
\hline \multirow{8}{*}{$6 \mathrm{H}$} & $49 \mathrm{RZ}$ & - & + & + & - & + & + & Enterococcus spp. \\
\hline & 50RZ & - & + & + & - & + & + & Enterococcus spp. \\
\hline & 51RZ & - & + & + & - & + & + & Enterococcus spp. \\
\hline & $52 \mathrm{RZ}$ & - & + & + & + & & & G.C \\
\hline & 53RZ & - & + & + & + & & & G.C \\
\hline & 54RZ & - & - & + & + & & & G.C \\
\hline & $55 \mathrm{RZ}$ & - & - & + & + & & & G.C \\
\hline & $56 \mathrm{RZ}$ & - & - & + & + & & & G.C \\
\hline \multirow{6}{*}{$6 \mathrm{~K}$} & $39 \mathrm{ks}$ & - & - & + & + & & & G.C \\
\hline & $40 \mathrm{ks}$ & - & - & + & + & & & G.C \\
\hline & $41 \mathrm{ks}$ & - & + & + & - & + & + & Enterococcus spp. \\
\hline & $42 \mathrm{ks}$ & - & + & + & - & + & - & Enterococcus spp. \\
\hline & $43 \mathrm{ks}$ & - & + & + & + & & & G.C \\
\hline & $44 \mathrm{ks}$ & - & + & + & - & + & + & Enterococcus spp. \\
\hline \multicolumn{9}{|c|}{ Sample 11} \\
\hline \multirow{8}{*}{$11 \mathrm{~L}$} & 64RZ & - & + & + & - & + & + & Enterococcus spp \\
\hline & $65 \mathrm{RZ}$ & - & - & + & + & & & G.C \\
\hline & $66 \mathrm{RZ}$ & - & + & - & - & - & - & Lactococcus spp. \\
\hline & $67 \mathrm{RZ}$ & - & - & + & + & & & G.C \\
\hline & $68 \mathrm{RZ}$ & - & + & + & + & & & G.C \\
\hline & 69RZ & - & - & + & + & & & G.C \\
\hline & $70 \mathrm{RZ}$ & - & + & + & + & & & G.C \\
\hline & $71 \mathrm{RZ}$ & - & - & + & + & & & G.C \\
\hline \multirow{5}{*}{$11 \mathrm{D}$} & $37 \mathrm{RZ}$ & - & + & - & - & - & - & Lactococcus spp. \\
\hline & $38 \mathrm{RZ}$ & - & + & + & - & + & + & Enterococcus spp. \\
\hline & 39RZ & - & - & + & + & & & G.C \\
\hline & $40 \mathrm{RZ}$ & - & - & + & + & & & G.C \\
\hline & $41 \mathrm{RZ}$ & - & - & + & + & & & G.C \\
\hline
\end{tabular}




\begin{tabular}{|c|c|c|c|c|c|c|c|c|}
\hline \multirow{4}{*}{ Samples } & \multirow{4}{*}{ isolates } & \multicolumn{6}{|c|}{ Pre - identification test } & \multirow{4}{*}{$\begin{array}{l}\text { Pre- } \\
\text { identification }\end{array}$} \\
\hline & & \multirow[t]{3}{*}{ Catalase } & \multirow{3}{*}{$\begin{array}{c}\begin{array}{c}\text { Growth } \\
\text { at }\end{array} \\
10^{\circ} \mathrm{C} \\
\end{array}$} & \multirow{3}{*}{$\begin{array}{c}\begin{array}{c}\text { Growth } \\
\text { at }\end{array} \\
45^{\circ} \mathrm{C} \\
\end{array}$} & \multirow{3}{*}{$\begin{array}{c}\mathrm{CO2} \\
\text { production } \\
\end{array}$} & \multirow{3}{*}{$\begin{array}{c}\text { SF } \\
\text { growth } \\
\end{array}$} & \multirow{3}{*}{$\begin{array}{l}\text { Growth } \\
\text { at } 6.5 \% \\
\mathrm{NaCl} \\
\end{array}$} & \\
\hline & & & & & & & & \\
\hline & & & & & & & & \\
\hline \multirow[t]{5}{*}{$11 \mathrm{D}$} & $42 \mathrm{RZ}$ & - & - & + & + & & & G.C \\
\hline & $43 \mathrm{RZ}$ & - & + & + & - & + & + & Enterococcus spp. \\
\hline & $72 \mathrm{RZ}$ & - & + & + & - & - & - & Lactocccus spp. \\
\hline & $73 \mathrm{RZ}$ & - & + & + & - & + & + & Enterococcus spp. \\
\hline & $74 \mathrm{RZ}$ & - & + & + & - & + & + & Enterococcus spp. \\
\hline \multirow[t]{5}{*}{$11 \mathrm{H}$} & $75 \mathrm{RZ}$ & - & + & + & + & & & G.C \\
\hline & $76 \mathrm{RZ}$ & - & + & + & + & & & G.C \\
\hline & $77 \mathrm{RZ}$ & - & + & + & + & & & G.C \\
\hline & $78 \mathrm{RZ}$ & - & - & + & + & & & G.C \\
\hline & $50 \mathrm{KS}$ & - & + & + & - & + & + & Enterococcus spp. \\
\hline \multirow[t]{2}{*}{$11 \mathrm{~K}$} & $51 \mathrm{KS}$ & - & + & + & - & + & + & Enterococcus spp. \\
\hline & $52 \mathrm{KS}$ & - & + & + & - & + & + & Enterococcus spp. \\
\hline \multicolumn{9}{|l|}{ Sample 29} \\
\hline & 94RZ & - & - & + & + & & & G.C \\
\hline \multirow[t]{6}{*}{$29 \mathrm{~L}$} & $95 \mathrm{RZ}$ & - & + & + & - & + & - & Enterococcus spp. \\
\hline & $97 \mathrm{RZ}$ & - & + & - & - & & & G.B \\
\hline & $98 \mathrm{RZ}$ & - & + & + & - & + & + & Enterococcus spp. \\
\hline & 99RZ & - & + & + & - & + & + & Enterococcus spp. \\
\hline & $100 \mathrm{RZ}$ & - & + & + & - & + & + & Enterococcus spp. \\
\hline & $101 \mathrm{RZ}$ & - & + & - & - & & & G.B \\
\hline \multirow[t]{9}{*}{$29 \mathrm{D}$} & $102 \mathrm{RZ}$ & - & + & + & + & & & G.C \\
\hline & $103 R Z$ & - & - & + & + & & & G.C \\
\hline & 104RZ & - & + & - & - & & & G.B \\
\hline & $105 \mathrm{RZ}$ & - & - & + & + & & & G.C \\
\hline & $106 \mathrm{RZ}$ & - & + & - & - & & & G.B \\
\hline & $107 \mathrm{RZ}$ & - & + & + & - & & & G.B \\
\hline & 219RZ & - & + & + & - & + & + & Enterococcus spp. \\
\hline & 220RZ & - & + & + & - & + & + & Enterococcus spp. \\
\hline & $221 \mathrm{RZ}$ & - & + & + & - & & & G.B \\
\hline \multirow[t]{6}{*}{$29 \mathrm{H}$} & $222 R Z$ & - & + & - & - & & & G.B \\
\hline & $223 R Z$ & - & - & + & + & & & G.C \\
\hline & $224 R Z$ & - & + & - & - & & & G.B \\
\hline & $225 \mathrm{RZ}$ & - & + & - & - & & & G.B \\
\hline & $226 \mathrm{RZ}$ & - & - & + & + & & & G.C \\
\hline & $64 \mathrm{KS}$ & - & + & + & - & + & + & Enterococcus spp. \\
\hline \multirow[t]{3}{*}{$29 \mathrm{~K}$} & $65 \mathrm{KS}$ & - & - & + & + & & & G.C \\
\hline & $66 \mathrm{KS}$ & - & - & + & + & & & G.C \\
\hline & $67 \mathrm{KS}$ & - & + & + & + & & & G.C \\
\hline \multicolumn{9}{|l|}{ Sample 63} \\
\hline $63 \mathrm{~L}$ & 134RZ & - & + & + & + & & & G.C \\
\hline
\end{tabular}

G: Group

Continuous 


\begin{tabular}{|c|c|c|c|c|c|c|c|c|}
\hline \multirow{4}{*}{ Samples } & \multirow{4}{*}{ Isolates } & \multicolumn{6}{|c|}{ Pre - identification test } & \multirow{4}{*}{ Pre- identification } \\
\hline & & \multirow[t]{3}{*}{ Catalase } & \multirow{3}{*}{$\begin{array}{c}\text { Growth } \\
\text { at } \\
10^{\circ} \mathrm{C} \\
\end{array}$} & \multirow{3}{*}{$\begin{array}{c}\text { Growth } \\
\text { at } \\
45^{\circ} \mathrm{C} \\
\end{array}$} & \multirow{3}{*}{$\begin{array}{c}\mathrm{CO}_{2} \\
\text { production }\end{array}$} & \multirow{3}{*}{$\begin{array}{l}\text { SF } \\
\text { growth }\end{array}$} & \multirow{3}{*}{$\begin{array}{l}\text { Growth } \\
\text { at } 6.5 \% \\
\mathrm{NaCl}\end{array}$} & \\
\hline & & & & & & & & \\
\hline & & & & & & & & \\
\hline & $135 \mathrm{RZ}$ & - & + & + & - & & & G.B \\
\hline & $136 \mathrm{RZ}$ & - & + & + & - & + & + & Enterococcus spp. \\
\hline \multirow[t]{6}{*}{$63 \mathrm{~L}$} & $137 \mathrm{RZ}$ & - & + & + & - & + & + & Enterococcus spp. \\
\hline & $138 \mathrm{RZ}$ & - & - & + & & & & G.A \\
\hline & 139RZ & - & - & + & & & & G.A \\
\hline & 140RZ & - & - & + & & & & G.A \\
\hline & $243 R Z$ & - & - & + & + & & & G.C \\
\hline & $244 \mathrm{RZ}$ & - & + & + & - & & & G.B \\
\hline \multirow[t]{7}{*}{$63 \mathrm{D}$} & $245 \mathrm{RZ}$ & - & + & + & - & + & + & Enterococcus spp. \\
\hline & $246 \mathrm{RZ}$ & - & + & + & - & + & + & Enterococcus spp. \\
\hline & $247 \mathrm{RZ}$ & - & - & + & - & & & G.A \\
\hline & $248 \mathrm{RZ}$ & - & - & + & - & 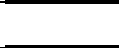 & 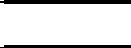 & G.A \\
\hline & $227 \mathrm{RZ}$ & - & + & + & - & + & + & Enterococcus spp. \\
\hline & $228 \mathrm{RZ}$ & - & + & + & - & + & + & Enterococcus spp. \\
\hline & $229 \mathrm{RZ}$ & - & + & + & - & & & G.B \\
\hline \multirow[t]{5}{*}{$63 \mathrm{H}$} & $230 \mathrm{RZ}$ & - & - & + & + & & & G.C \\
\hline & $231 \mathrm{RZ}$ & - & + & - & - & & & G.B \\
\hline & $232 \mathrm{RZ}$ & - & - & + & - & & & G.A \\
\hline & $233 \mathrm{RZ}$ & - & + & + & - & + & + & Enterococcus spp. \\
\hline & $234 \mathrm{RZ}$ & - & - & + & + & & & G.C \\
\hline \multirow[t]{2}{*}{$63 \mathrm{~K}$} & $71 \mathrm{KS}$ & - & + & + & - & + & + & Enterococcus spp. \\
\hline & $72 \mathrm{KS}$ & - & - & + & + & & & G.C \\
\hline \multicolumn{9}{|l|}{ Sample 64} \\
\hline & 249RZ & - & + & + & - & + & + & Enterococcus spp. \\
\hline & $250 \mathrm{RZ}$ & - & + & + & - & + & + & Enterococcus spp. \\
\hline \multirow[t]{5}{*}{$64 \mathrm{~L}$} & $251 \mathrm{RZ}$ & - & + & - & + & & 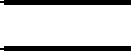 & G.C \\
\hline & $252 \mathrm{RZ}$ & - & + & + & - & 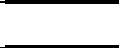 & & G.B \\
\hline & $253 \mathrm{RZ}$ & - & + & - & - & & & G.C \\
\hline & 254RZ & - & + & + & - & + & + & Enterococcus spp. \\
\hline & $255 \mathrm{RZ}$ & - & + & + & - & + & + & Enterococcus spp. \\
\hline \multirow[t]{5}{*}{$64 \mathrm{D}$} & $256 \mathrm{RZ}$ & - & + & + & - & + & + & Enterococcus spp. \\
\hline & $257 \mathrm{RZ}$ & - & + & - & - & & & G.B \\
\hline & $258 \mathrm{RZ}$ & - & + & + & - & & & G.B \\
\hline & $259 \mathrm{RZ}$ & - & + & - & - & & & G.B \\
\hline & $281 \mathrm{RZ}$ & - & + & + & - & & & G.B \\
\hline \multirow[t]{3}{*}{$64 \mathrm{H}$} & $282 \mathrm{RZ}$ & - & + & + & - & 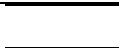 & 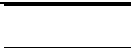 & G.B \\
\hline & $283 R Z$ & - & + & + & - & + & + & Enterococcus spp. \\
\hline & $68 \mathrm{KS}$ & - & + & + & - & + & + & Enterococcus spp. \\
\hline \multirow[t]{2}{*}{$64 \mathrm{~K}$} & $69 \mathrm{KS}$ & 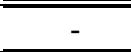 & + & + & - & + & + & Enterococcus spp. \\
\hline & $70 \mathrm{KS}$ & - & + & + & - & & & G.B \\
\hline \multicolumn{9}{|l|}{ Sample 74} \\
\hline $74 \mathrm{~L}$ & 141RZ & - & + & + & - & + & + & Enterococcus spp. \\
\hline
\end{tabular}




\begin{tabular}{|c|c|c|c|c|c|c|c|c|}
\hline \multirow{4}{*}{ Samples } & \multirow{4}{*}{ Isolates } & \multicolumn{6}{|c|}{ Pre - identification test } & \multirow{4}{*}{ Pre- identification } \\
\hline & & \multirow[t]{3}{*}{ Catalase } & \multirow{3}{*}{$\begin{array}{c}\text { Growth } \\
\text { at } \\
10^{\circ} \mathrm{C}\end{array}$} & \multirow{3}{*}{$\begin{array}{c}\begin{array}{c}\text { Growth } \\
\text { at }\end{array} \\
45^{\circ} \mathrm{C} \\
\end{array}$} & \multirow{3}{*}{$\begin{array}{c}\mathrm{CO}_{2} \\
\text { production } \\
\end{array}$} & \multirow{3}{*}{$\begin{array}{l}\text { SF } \\
\text { growth } \\
\end{array}$} & \multirow{3}{*}{$\begin{array}{l}\text { Growth } \\
\text { at } 6.5 \% \\
\mathrm{NaCl}\end{array}$} & \\
\hline & & & & & & & & \\
\hline & & & & & & & & \\
\hline \multirow[t]{6}{*}{$74 \mathrm{~L}$} & $142 \mathrm{RZ}$ & - & + & + & - & + & + & Enterococcus spp. \\
\hline & $143 \mathrm{RZ}$ & - & - & + & + & & & G.C \\
\hline & 144RZ & - & + & + & + & & & G.C \\
\hline & $145 \mathrm{RZ}$ & - & + & - & - & & & G.B \\
\hline & 146RZ & - & - & + & + & & & G.C \\
\hline & 147RZ & - & - & + & + & & & G.C \\
\hline \multirow[t]{7}{*}{$74 \mathrm{D}$} & $236 \mathrm{RZ}$ & - & + & + & - & + & + & Enterococcus spp. \\
\hline & $237 \mathrm{RZ}$ & - & + & + & - & + & + & Enterococcus spp. \\
\hline & $238 \mathrm{RZ}$ & - & + & + & - & + & + & Enterococcus spp. \\
\hline & $239 \mathrm{RZ}$ & - & - & + & + & & & G.C \\
\hline & 240RZ & - & + & - & - & & & G.B \\
\hline & 241RZ & - & - & + & + & & & G.C \\
\hline & $242 \mathrm{RZ}$ & - & - & + & + & & & G.C \\
\hline \multirow[t]{8}{*}{$74 \mathrm{H}$} & $148 \mathrm{RZ}$ & - & - & + & - & + & + & Enterococcus spp. \\
\hline & 149RZ & - & + & + & + & & & G.C \\
\hline & 150RZ & - & - & + & - & + & - & Enterococcus spp. \\
\hline & 151RZ & - & + & + & + & & & G.C \\
\hline & $152 \mathrm{RZ}$ & - & + & + & + & & & G.C \\
\hline & $153 \mathrm{RZ}$ & - & + & + & + & & . & G.C \\
\hline & $58 \mathrm{KS}$ & - & + & + & - & + & + & Enterococcus spp. \\
\hline & $59 \mathrm{KS}$ & - & + & + & - & + & - & Enterococcus spp. \\
\hline \multirow[t]{4}{*}{$74 K$} & $60 \mathrm{KS}$ & - & + & + & + & + & - & Enterococcus spp. \\
\hline & $61 \mathrm{KS}$ & - & + & + & + & & & G.C \\
\hline & $62 \mathrm{KS}$ & - & + & + & + & & & G.C \\
\hline & $63 \mathrm{KS}$ & - & - & + & + & & & G.C \\
\hline \multicolumn{9}{|l|}{ Sample 76} \\
\hline & 260RZ & - & + & + & - & + & + & Enterococcus spp. \\
\hline & 261RZ & - & + & + & - & + & + & Enterococcus spp. \\
\hline & $262 \mathrm{RZ}$ & - & + & + & - & + & + & Enterococcus spp. \\
\hline \multirow[t]{7}{*}{$76 \mathrm{~L}$} & $263 R Z$ & - & + & - & + & & & G.C \\
\hline & 264RZ & - & + & - & - & & & G.B \\
\hline & $265 \mathrm{RZ}$ & - & - & + & + & & & G.C \\
\hline & $266 \mathrm{RZ}$ & - & + & - & + & & & G.C \\
\hline & 274RZ & - & - & + & + & & & G.C \\
\hline & $275 \mathrm{RZ}$ & - & - & + & + & & & G.C \\
\hline & $276 \mathrm{RZ}$ & - & - & + & + & 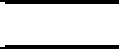 & & G.C \\
\hline \multirow[t]{5}{*}{$76 \mathrm{D}$} & $277 \mathrm{RZ}$ & - & + & + & - & + & + & Enterococcus spp. \\
\hline & $278 \mathrm{RZ}$ & - & + & + & - & + & + & Enterococcus spp. \\
\hline & 279RZ & - & + & + & - & + & + & Enterococcus spp. \\
\hline & $280 \mathrm{RZ}$ & - & + & + & - & & & G.B \\
\hline & 284RZ & - & + & + & - & & & G.B \\
\hline
\end{tabular}

G:Group

Continuous 


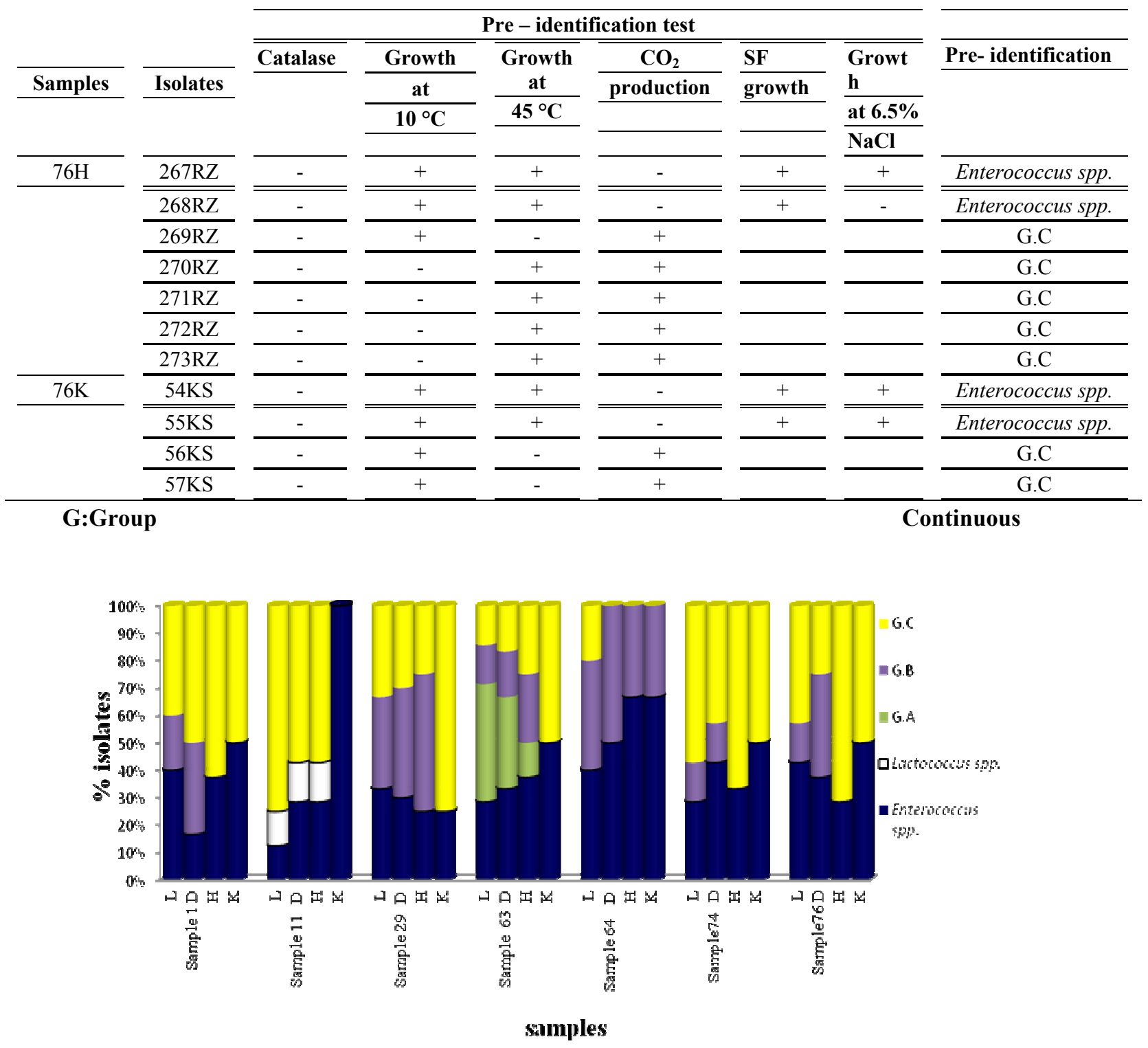

Fig. 1. Lactic acid bacteria diversity during Egyptian Kishk manufacturing (L) refers to Laban Zeer; (D) Dough; (H) Hama and (K) Kishk

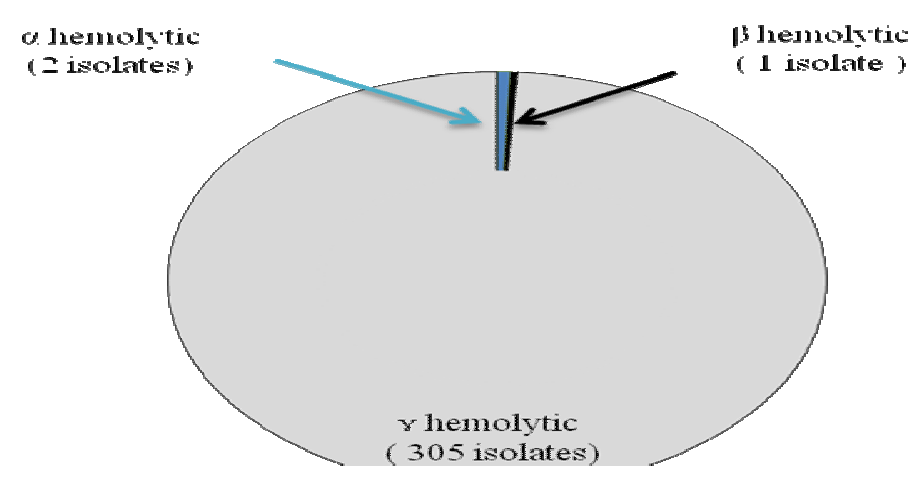


Fig. 2. Hemolytic activity of Lactic acid bacteria isolates 
Group A isolates; The taste and odor of the curd were pleasant, The curds of fermented milk were smooth, compact and without syneresis when the isolates (23RZ, 86RZ, 162RZ, 182RZ and 207RZ) were used in fermentation, while the syneresis was noted with the both (232RZ and 27KS) isolates. Most isolates produced the bitterness defect with varying degrees in fermented milk, this is refer to high proteolytic activity of tested isolates. It is important to bear in mind that highly proteolytic strains are not always the most suitable for use as starter cultures, since excessive proteolysis can cause uncontrolled production of bitter peptides and other undesirable compounds, or even excessive casein hydrolysis resulting in a too-soft final product (Buffa et al., 2005). Results in Table 4 were referred to sensorial evaluation means of group A isolates.

Group B isolates; they were divided into four different tastes. Firstly, the taste was flat. Secondly, the curd had bitterness taste for example but not limited to (225RZ, 231RZ, 240RZand 244RZ).Thirdly, the curd was unpleasant taste like (106RZ, 160RZ AND 170 RZ). Finally, the curd was pleasant taste and good odor without any defects such as (101RZ, 157RZ, 159RZ, 172RZ, 190RZ, $281 \mathrm{RZ}$ and 284RZ). It is observed in Tables ( 3 and 5) that there were significant differences $(\mathrm{p} \geq 0.05)$ between panelists in overall acceptance.

Table 4. Sensorial evaluation means of fermented milk by isolates (group A)

\begin{tabular}{cccccccc}
\hline \multirow{2}{*}{ Isolates } & \multicolumn{7}{c}{ Mean } \\
\cline { 2 - 8 } & Color(10) & Taste (10) & Smell(10) & Texture (10) & Appearance(10) & Total (50) & Over all \\
\hline $23 \mathrm{RZ}$ & 8.66 & 8.86 & 9 & 7.73 & 8.53 & 42.8 & 2 \\
\hline $86 \mathrm{RZ}$ & 9.23 & 9 & 9 & 8.93 & 8.93 & 45.1 & 2 \\
\hline $87 \mathrm{RZ}$ & 8.00 & 2.33 & 5.13 & 6.66 & 7.06 & 29.2 & 1 \\
\hline $115 \mathrm{RZ}$ & 5.60 & 3.06 & 1.93 & 3.20 & 3.33 & 17.13 & 1 \\
\hline $116 \mathrm{RZ}$ & 4.86 & 3 & 2 & 2.86 & 3 & 15.73 & 1 \\
\hline $122 \mathrm{RZ}$ & 5.60 & 3.06 & 2.13 & 2.33 & 2.33 & 15.46 & 1 \\
\hline $127 \mathrm{RZ}$ & 5.20 & 3.4 & 3.06 & 2.46 & 2.33 & 16.46 & 1 \\
\hline $130 \mathrm{RZ}$ & 5.33 & 3.46 & 3.13 & 2.20 & 2.20 & 16.33 & 1 \\
\hline $138 \mathrm{RZ}$ & 4.43 & 2.80 & 2.46 & 2.26 & 2.53 & 14.5 & 1 \\
\hline $139 \mathrm{RZ}$ & 2.80 & 2.26 & 1.80 & 1.80 & 1.73 & 10.26 & 1 \\
\hline $140 \mathrm{RZ}$ & 3.93 & 2.80 & 2.13 & 1.93 & 1.86 & 12.8 & 1 \\
\hline $154 \mathrm{RZ}$ & 5.26 & 3.33 & 2.73 & 2.93 & 3 & 17.25 & 1 \\
\hline $161 \mathrm{RZ}$ & 5.53 & 3.6 & 2.86 & 2.73 & 2.86 & 17.60 & 1 \\
\hline $162 \mathrm{RZ}$ & 8.06 & 8.86 & 8.66 & 8.26 & 7.90 & 41.76 & 2 \\
\hline $163 \mathrm{RZ}$ & 7.86 & 6.93 & 2.06 & 7.46 & 7.46 & 31.80 & 1 \\
\hline $164 \mathrm{RZ}$ & 4.93 & 3.06 & 2.66 & 2.80 & 2.93 & 16.40 & 1 \\
\hline $182 \mathrm{RZ}$ & 9.20 & 8.5 & 8.66 & 8.93 & 9.03 & 44.40 & 2 \\
\hline $184 \mathrm{RZ}$ & 5.20 & 2.86 & 2.20 & 3.33 & 3.33 & 16.93 & 1 \\
\hline $191 \mathrm{RZ}$ & 5.33 & 2.86 & 2.66 & 3 & 2.86 & 16.73 & 1 \\
\hline $202 \mathrm{RZ}$ & 4.40 & 2.4 & 2.33 & 2.13 & 2.20 & 13.46 & 1 \\
\hline $207 \mathrm{RZ}$ & 8.66 & 8.9 & 8.9 & 8.86 & 8.56 & 43.93 & 2 \\
\hline $208 \mathrm{RZ}$ & 5.40 & 2.86 & 2.60 & 2.73 & 2.73 & 16.33 & 1 \\
\hline $211 \mathrm{RZ}$ & 4.86 & 3.2 & 2.20 & 1.60 & 1.66 & 13.53 & 1 \\
\hline $212 \mathrm{RZ}$ & 4.93 & 3.2 & 2.33 & 1.86 & 1.73 & 14.06 & 1 \\
\hline $213 \mathrm{RZ}$ & 4.06 & 2.8 & 2.13 & 2 & 1.86 & 12.86 & 1 \\
\hline $232 \mathrm{RZ}$ & 5.53 & 3.06 & 2.13 & 1.93 & 1.73 & 14.40 & 1 \\
\hline $247 \mathrm{RZ}$ & 5.53 & 4.86 & 3.40 & 2.93 & 1.80 & 18.53 & 1 \\
\hline $248 \mathrm{RZZ}$ & 5.60 & 4.20 & 3.06 & 1.80 & 1.66 & 15.40 & 1 \\
\hline $27 \mathrm{~K} \mathrm{n}$ & 5.20 & 3.40 & 2.40 & 1.86 & 1.80 & 14.66 & 1 \\
\hline $29 \mathrm{KS}$ & 5.06 & 3.6 & 2.73 & 2.06 & 1.80 & 15.26 & 1 \\
\hline Mean & 5.81 & 4.22 & 3.61 & 3.72 & 3.69 & 21.03 & 1.16 \\
\hline & & & & & & & \\
\hline
\end{tabular}


$\mathrm{LSD}_{0.01} \quad 0.84$

0.76

0.62

0.71

0.66

2.26

Overall 1: unacceptable

Overall 2: acceptable

Table 5. Sensorial evaluation means of fermented milk by isolates (group B)

\begin{tabular}{|c|c|c|c|c|c|c|c|}
\hline \multirow{2}{*}{ Isolates } & \multicolumn{7}{|c|}{ Mean } \\
\hline & Color (10) & Taste (10) & Smell (10) & Texture (10) & Appearance (10) & Total (50) & Over all \\
\hline $17 \mathrm{RZ}$ & 7.40 & 2.93 & 4.06 & 1.33 & 1.86 & 17.60 & 1 \\
\hline $27 \mathrm{RZ}$ & 7.9 & 5.13 & 5.13 & 6.8 & 6.86 & 31.86 & 1 \\
\hline $28 \mathrm{RZ}$ & 7.9 & 5.13 & 5.13 & 6.8 & 6.86 & 31.86 & 1 \\
\hline $46 \mathrm{RZ}$ & 8 & 7.06 & 6.60 & 5.13 & 6 & 32.8 & 1 \\
\hline 97RZ & 8 & 3.53 & 3.26 & 5.20 & 5.40 & 25.4 & 1 \\
\hline $101 \mathrm{RZ}$ & 9 & 8.1 & 7.40 & 8.6 & 8.60 & 41.7 & 2 \\
\hline 104RZ & 8 & 1 & 1.46 & 4.53 & 4.53 & 19.53 & 1 \\
\hline 106RZ & 8 & 1.13 & 1.46 & 4.46 & 4.53 & 19.60 & 1 \\
\hline $107 \mathrm{RZ}$ & 8 & 5.96 & 5.53 & 6.53 & 6.60 & 32.63 & 1.06 \\
\hline $120 \mathrm{RZ}$ & 5 & 3.06 & 2.20 & 2.13 & 1.93 & 14.33 & 1 \\
\hline 131RZ & 5.46 & 3 & 2.40 & 2.93 & 2.93 & 16.73 & 1 \\
\hline 132RZ & 3.20 & 1.53 & 1.26 & 0.53 & 1.40 & 7.93 & 1 \\
\hline $133 \mathrm{RZ}$ & 5.73 & 3 & 2.40 & 2.80 & 2.66 & 16.60 & 1 \\
\hline 135RZ & 7.66 & 5.43 & 5.73 & 6.7 & 6.46 & 32.03 & 1.26 \\
\hline $145 \mathrm{RZ}$ & 4.53 & 3.2 & 2.86 & 2.80 & 2.66 & 16.0 & 1 \\
\hline 156RZ & 7.40 & 3.20 & 3.40 & 5.13 & 5.33 & 24.46 & 1 \\
\hline $157 \mathrm{RZ}$ & 8.86 & 8.1 & 8.46 & 7.73 & 7.80 & 41 & 2 \\
\hline 158RZ & 7.60 & 1.93 & 2.73 & 4.53 & 5.26 & 22.26 & 1 \\
\hline 159RZ & 8 & 7.8 & 7.53 & 6.6 & 7.36 & 37.36 & 2 \\
\hline 160RZ & 4.53 & 3.06 & 2.86 & 2.60 & 2.60 & 15.66 & 1 \\
\hline 170RZ & 5.20 & 2.80 & 2 & 3.46 & 3.46 & 16.93 & 1 \\
\hline 171RZ & 4.53 & 1.80 & 1.73 & 1.46 & 1.73 & 11.26 & 1 \\
\hline 172RZ & 9 & 8.9 & 8.86 & 8.9 & 8.66 & 44.36 & 2 \\
\hline $175 \mathrm{RZ}$ & 4 & 0.53 & 0.66 & 1.06 & 1.26 & 7.53 & 1 \\
\hline 176RZ & 7.86 & 5.06 & 5.26 & 6.33 & 6.46 & 31 & 1.06 \\
\hline 190RZ & 8 & 7.06 & 7.83 & 8.8 & 8.86 & 40.56 & 2 \\
\hline 192RZ & 5.53 & 3 & 2.26 & 2.66 & 2.73 & 16.20 & 1 \\
\hline 201RZ & 5.13 & 3.20 & 2.20 & 2.40 & 2.33 & 15.26 & 1 \\
\hline $203 R Z$ & 6.73 & 4.8 & 5.26 & 5.66 & 5.13 & 27.60 & 1 \\
\hline 209RZ & 5.40 & 3.13 & 3 & 2.13 & 2.06 & 15.73 & 1 \\
\hline 210RZ & 4.73 & 2.93 & 2.26 & 2.33 & 2.20 & 14.46 & 1 \\
\hline 221RZ & 4.60 & 3.06 & 2.20 & 1.60 & 1.60 & 13.06 & 1 \\
\hline $222 \mathrm{RZ}$ & 5.26 & 3.40 & 2.33 & 1.93 & 1.93 & 14.86 & 1 \\
\hline 224RZ & 4.73 & 3 & 2.26 & 1.80 & 1.66 & 13.46 & 1 \\
\hline $225 \mathrm{RZ}$ & 5.46 & 3.66 & 2.60 & 2.20 & 2 & 15.93 & 1 \\
\hline 229RZ & 5.73 & 3.26 & 2.40 & 1.86 & 1.73 & 15 & 1 \\
\hline 231RZ & 4.6 & 2.93 & 2.33 & 2.13 & 1.93 & 14 & 1 \\
\hline 240RZ & 5.13 & 2.80 & 2.13 & 4.80 & 4.40 & 19.26 & 1 \\
\hline 244RZ & 4.53 & 3.06 & 2.86 & 2.60 & 2.60 & 15.66 & 1 \\
\hline 252RZ & 7 & 5 & 6.06 & 6.20 & 5.86 & 30.13 & 1.3 \\
\hline 253RZ & 5.26 & 3.26 & 2.73 & 2.93 & 2.93 & 17.13 & 1 \\
\hline $257 \mathrm{RZ}$ & 7 & 5.4 & 6.20 & 6.40 & 6.33 & 31.33 & 1.3 \\
\hline 258RZ & 5.13 & 3.20 & 2.33 & 2.06 & 1.80 & 14.53 & 1 \\
\hline 259RZ & 5.26 & 3.20 & 2.53 & 2.26 & 2.13 & 15.40 & 1 \\
\hline
\end{tabular}


Con. Table 5.

\begin{tabular}{cccccccc}
\hline \multirow{2}{*}{ Isolates } & \multicolumn{7}{c}{ Mean } \\
\cline { 2 - 8 } & Color (10) & Taste (10) & Smell (10) & $\begin{array}{c}\text { Texture(10 } \\
\text { ) }\end{array}$ & $\begin{array}{c}\text { Appearance(10 } \\
\text { ) }\end{array}$ & Total (50) & Over all \\
\hline $264 \mathrm{RZ}$ & 7.46 & 4.56 & 5.80 & 5.80 & 6.06 & 29.70 & 1 \\
\hline $280 \mathrm{RZ}$ & 8.20 & 4.73 & 4.66 & 4.80 & 4.60 & 27 & 1.13 \\
\hline $281 \mathrm{RZ}$ & 7.53 & 7.6 & 8.36 & 7.26 & 8.06 & 38.90 & 2 \\
\hline $282 \mathrm{RZ}$ & 7 & 4.50 & 6.23 & 5.66 & 5.80 & 29.20 & 1.2 \\
\hline $284 \mathrm{RZ}$ & 7.60 & 7.9 & 7.33 & 7.26 & 7.93 & 38.1 & 2 \\
\hline $31 \mathrm{KS}$ & 4.40 & 2.80 & 2.06 & 1.73 & 1.66 & 12.66 & 1 \\
\hline $32 \mathrm{KS}$ & 5.26 & 3.06 & 2.13 & 2 & 2 & 14.46 & 1 \\
\hline $45 \mathrm{KS}$ & 5.06 & 2.80 & 1.93 & 1.93 & 1.80 & 13.53 & 1 \\
\hline $70 \mathrm{KS}$ & 4.66 & 3.26 & 2.40 & 1.80 & 1.80 & 13.93 & 1 \\
\hline Mean & 6.29 & 4.02 & 3.83 & 4.08 & 4.13 & 22.37 & 1.17 \\
\hline LSD & 0.72 & 0.88 & 0.73 & 0.72 & 0.64 & 2.48 & - \\
\hline Overall 1: non acceptable & & Overall 2. &
\end{tabular}

Overall 1: non acceptable Overall 2: acceptable

Overall: $2>$ overall $1>1$; refer to different overall acceptability between panelists

Table 6. Sensorial evaluation means of fermented milk by isolates (group C)

\begin{tabular}{|c|c|c|c|c|c|c|c|}
\hline \multirow[b]{2}{*}{ Isolates } & \multicolumn{7}{|c|}{ Mean } \\
\hline & Color(10) & Taste(10) & Smell (10) & $\begin{array}{c}\text { Texture(10 } \\
)\end{array}$ & $\begin{array}{c}\text { Appearance(10 } \\
)\end{array}$ & Total(50) & Over all \\
\hline $16 \mathrm{RZ}$ & 7.40 & 2.93 & 4.06 & 6.46 & 4.26 & 25.13 & 1 \\
\hline $20 \mathrm{RZ}$ & 7.53 & 2.93 & 3.66 & 6.40 & 4.66 & 25.20 & 1 \\
\hline $21 \mathrm{RZ}$ & 5.93 & 2.00 & 1.66 & 5.93 & 5.93 & 21.48 & 1 \\
\hline $22 \mathrm{RZ}$ & 7.60 & 0.80 & 1.06 & 7.00 & 5.53 & 22.00 & 1 \\
\hline $24 \mathrm{RZ}$ & 7.66 & 6 & 5.73 & 6.00 & 5.60 & 31.00 & 1 \\
\hline $25 \mathrm{RZ}$ & 7.93 & 5.40 & 4.73 & 5.93 & 6.26 & 30.26 & 1.20 \\
\hline $31 \mathrm{RZ}$ & 7.33 & 5.80 & 5.86 & 5.86 & 5.93 & 30.800 & 1.13 \\
\hline $32 \mathrm{RZ}$ & 7.73 & 4.66 & 4.40 & 7.40 & 6.46 & 30.66 & 1 \\
\hline $34 \mathrm{RZ}$ & 7.40 & 3.40 & 3.26 & 3.73 & 3.60 & 21.40 & 1 \\
\hline 39RZ & 8.60 & 3.93 & 2.73 & 4.93 & 4.93 & 25.13 & 1 \\
\hline $40 \mathrm{RZ}$ & 8 & 2.00 & 1.26 & 0.00 & 0.00 & 11.26 & 1 \\
\hline $41 \mathrm{RZ}$ & 7.80 & 4.46 & 4.06 & 5.93 & 6.26 & 28.53 & 1 \\
\hline 42RZ & 7.60 & 2.40 & 2.86 & 4.93 & 4.93 & 22.73 & 1 \\
\hline $47 \mathrm{RZ}$ & 7.80 & 4.53 & 4.86 & 3.60 & 3.60 & 24.40 & 1.13 \\
\hline 48RZ & 8 & 3.66 & 3.93 & 6.66 & 6.40 & 28.66 & 1 \\
\hline 52RZ & 8 & 4.26 & 3.20 & 6.06 & 6.20 & 27.73 & 1 \\
\hline 53RZ & 8 & 4.26 & 3.20 & 6.40 & 6.33 & 28.20 & 1 \\
\hline 54RZ & 7.93 & 5.26 & 5.13 & 4.13 & 4.26 & 26.73 & 1 \\
\hline $55 \mathrm{RZ}$ & 7.53 & 4.90 & 4.66 & 4.66 & 4.86 & 26.63 & 1 \\
\hline 56RZ & 7.60 & 4.46 & 4.53 & 4.13 & 4.26 & 25.00 & 1 \\
\hline 61RZ & 5.40 & 2.13 & 2.86 & 4.33 & 4.13 & 26.13 & 1 \\
\hline 62RZ & 6.26 & 2.60 & 5.33 & 5.80 & 5.06 & 25.06 & 1 \\
\hline 63RZ & 7.53 & 4.36 & 3.46 & 1.40 & 1.80 & 18.56 & 1 \\
\hline $65 \mathrm{RZ}$ & 6.26 & 2.66 & 5.33 & 5.80 & 5.06 & 25.13 & 1 \\
\hline 67RZ & 7.20 & 1.46 & 3.53 & 6.00 & 5.93 & 24.13 & 1 \\
\hline 68RZ & 4.93 & 0.86 & 0.86 & 3.00 & 2.60 & 12.26 & 1 \\
\hline 69RZ & 5.73 & 2.40 & 1.86 & 3.60 & 2.93 & 16.53 & 1 \\
\hline 70RZ & 6.13 & 1.73 & 1.00 & 4.86 & 4.73 & 18.46 & 1 \\
\hline
\end{tabular}


71RZ $\quad 7.2$

Overall 1: non acceptable

Overall: $2>$ overall $1>1$; refer to different overall acceptability between panelists

Con. Table 6.

\begin{tabular}{|c|c|c|c|c|c|c|c|}
\hline \multirow[b]{2}{*}{ Isolates } & \multicolumn{7}{|c|}{ Mean } \\
\hline & Color (10) & $\begin{array}{c}\text { Taste } \\
\text { (10) }\end{array}$ & $\begin{array}{c}\text { Smell } \\
(10)\end{array}$ & $\begin{array}{c}\text { Texture } \\
\text { (10) }\end{array}$ & $\begin{array}{c}\text { Appearance } \\
\text { (10) }\end{array}$ & $\begin{array}{l}\text { Total } \\
\text { (50) }\end{array}$ & Over all \\
\hline $75 \mathrm{RZ}$ & 5.13 & 3.26 & 3.53 & 2.13 & 2.20 & 15.73 & 1 \\
\hline 76RZ & 5.06 & 3.26 & 3.33 & 2.53 & 2.66 & 16.86 & 1 \\
\hline $77 \mathrm{RZ}$ & 7.13 & 1.53 & 2.33 & 3.73 & 3.60 & 18.53 & 1 \\
\hline $78 \mathrm{RZ}$ & 6.20 & 2.33 & 3.06 & 3.46 & 3.46 & 18.52 & 1 \\
\hline 79RZ & 6.06 & 2.53 & 2.80 & 2.93 & 3.13 & 17.46 & 1 \\
\hline $80 \mathrm{RZ}$ & 5.33 & 2.86 & 2.53 & 2.40 & 2.40 & 15.53 & 1 \\
\hline 81RZ & 6 & 2.26 & 2.53 & $2 . .80$ & 2.53 & 16.13 & 1 \\
\hline 84RZ & 5.2 & 2.80 & 2.06 & 1.60 & 2.60 & 13.26 & 1 \\
\hline $88 \mathrm{RZ}$ & 5.26 & 2.73 & 2.33 & 2.20 & 2.20 & 14.73 & 1 \\
\hline 91RZ & 5.73 & 3.13 & 2.53 & 1.93 & 1.86 & 15.20 & 1 \\
\hline 92RZ & 5.26 & 2.86 & 2.13 & 1.80 & 1.73 & 13.86 & 1 \\
\hline 93RZ & 6.73 & 5.66 & 4.96 & 6.46 & 6.73 & 30.56 & 1 \\
\hline 94RZ & 5.26 & 2.80 & 2.80 & 2.66 & 2.404 & 15.93 & 1 \\
\hline 128RZ & 4.40 & 2.26 & 2.40 & 2.26 & 2.13 & 13.46 & 1 \\
\hline $129 \mathrm{RZ}$ & 5.26 & 2.80 & 2.80 & 2.06 & 2.40 & 15.93 & 1 \\
\hline 134RZ & 5.06 & 3.00 & 2.73 & 3.13 & 3.00 & 16.93 & 1 \\
\hline 143RZ & 4.66 & 4 & 3 & 2.73 & 2.66 & 17.06 & 1 \\
\hline 144RZ & 5.66 & 3.33 & 2.60 & 2.93 & 3.00 & 17.53 & 1 \\
\hline $146 \mathrm{RZ}$ & 5.06 & 3.33 & 2.60 & 2.66 & 2.80 & 16.46 & 1 \\
\hline 147RZ & 4.20 & 2.93 & 2.40 & 2.13 & 2.13 & 13.80 & 1 \\
\hline 149RZ & 5.26 & 2.80 & 2.80 & 2.66 & 2.40 & 15.93 & 1 \\
\hline 151RZ & 4.80 & 3.46 & 2.93 & 2.33 & 2.33 & 15.86 & 1 \\
\hline $152 \mathrm{RZ}$ & 5.26 & 3.46 & 2.86 & 2.40 & 2.46 & 16.46 & 1 \\
\hline $153 \mathrm{RZ}$ & 5.06 & 3.53 & 2.93 & 2.86 & 2.53 & 16.93 & 1 \\
\hline $165 \mathrm{RZ}$ & 4.60 & 2.40 & 1.93 & 1.73 & 2.06 & 12.73 & 1 \\
\hline 166RZ & 4.73 & 2.60 & 4.73 & 2.06 & 2.33 & 14.00 & 1 \\
\hline 169RZ & 5 & 3.13 & 2.60 & 2.40 & 2.26 & 15.40 & 1 \\
\hline 174RZ & 5.26 & 3.26 & 2.73 & 2.93 & 2.93 & 17.13 & 1 \\
\hline 178RZ & 4 & 2.80 & 2.40 & 2.40 & 2.20 & 13.80 & 1 \\
\hline 179RZ & 4.73 & 2.80 & 2.13 & 2.06 & 2.33 & 14.06 & 1 \\
\hline 180RZ & 4.73 & 3.06 & 2.40 & 2.26 & 2.33 & 14.80 & 1 \\
\hline 181RZ & 5.73 & 2.93 & 1.93 & 2.93 & 3.00 & 16.53 & 1 \\
\hline 183RZ & 4.86 & 2.93 & 2.33 & 2.20 & 2.46 & 14.80 & 1 \\
\hline 185RZ & 5.53 & 2.93 & 2.33 & 3.46 & 3.46 & 17.73 & 1 \\
\hline 186RZ & 5 & 3.26 & 2.53 & 2.53 & 2.60 & 15.86 & 1 \\
\hline $187 \mathrm{RZ}$ & 4.66 & 2.93 & 2.33 & 2.40 & 2.20 & 14.53 & 1 \\
\hline 188RZ & 4.66 & 2.93 & 2.33 & 2.40 & 2.20 & 14.53 & 1 \\
\hline 189RZ & 4.66 & 2.93 & 2.26 & 2.46 & 2.40 & 14.73 & 1 \\
\hline 193RZ & 4.86 & 3.40 & 2.80 & 1.73 & 1.60 & 14.40 & 1 \\
\hline 194RZ & 5.33 & 2.93 & 2.13 & 2.53 & 2.66 & 15.60 & 1 \\
\hline 195RZ & 4.60 & 3.20 & 2.40 & 1.73 & 1.80 & 13.73 & 1 \\
\hline 196RZ & 4.40 & 2.86 & 2.26 & 2.26 & 2.26 & 14.13 & 1 \\
\hline 197RZ & 4.46 & 3.06 & 2.26 & 2.00 & 2.06 & 13.86 & 1 \\
\hline 198RZ & 5.2 & 3.06 & 2.26 & 2.80 & 2.80 & 16.13 & 1 \\
\hline 199RZ & 5.26 & 3.40 & 2.33 & 1.93 & 1.93 & 14.86 & 1 \\
\hline 200RZ & 4.80 & 3.06 & 2.46 & 2.60 & 2.66 & 15.60 & 1 \\
\hline 204RZ & 5.33 & 3.20 & 2.66 & 2.66 & 2.80 & 16.66 & 1 \\
\hline
\end{tabular}


Overall 1: non acceptable

Overall 1: non acceptable Overall 2: acceptable

Overall; $2>$ overall $>1$; refer to different overall acceptability between panelists

Con. Table 6.

\begin{tabular}{|c|c|c|c|c|c|c|c|}
\hline \multirow{2}{*}{ Isolates } & \multicolumn{7}{|c|}{ Mean } \\
\hline & Color (10) & Taste (10) & Smell (10) & Texture (10) & Appearance (10) & Total (50) & Over all \\
\hline $205 \mathrm{RZ}$ & 5.60 & 3.33 & 2.26 & 1.86 & 1.93 & 15.00 & 1 \\
\hline 206RZ & 5.06 & 3.20 & 2.53 & 2.06 & 1.93 & 14.80 & 1 \\
\hline 214RZ & 5.13 & 3.13 & 2.46 & 2.06 & 2.00 & 14.80 & 1 \\
\hline 216RZ & 5.73 & 3.66 & 2.66 & 2.00 & 2.06 & 16.20 & 1 \\
\hline $217 \mathrm{RZ}$ & 4.73 & 3.06 & 2.26 & 2.00 & 1.93 & 14.00 & 1 \\
\hline 218RZ & 5.40 & 3.26 & 2.60 & 1.73 & 1.60 & 14.60 & 1 \\
\hline 223RZ & 5.26 & 3.20 & 2.46 & 2.00 & 2.00 & 14.93 & 1 \\
\hline 226RZ & 5.53 & 3.46 & 2.33 & 2.26 & 2.13 & 15.73 & 1 \\
\hline 230RZ & 5.06 & 3.26 & 2.60 & 2.06 & 2.13 & 15.13 & 1 \\
\hline 234RZ & 5.26 & 3.40 & 2.33 & 1.86 & 1.80 & 14.66 & 1 \\
\hline 239RZ & 5.26 & 3.26 & 2.73 & 2.73 & 2.73 & 16.73 & 1 \\
\hline 241RZ & 4.93 & 2.73 & 1.80 & 1.80 & 1.73 & 13.00 & 1 \\
\hline 242RZ & 4.73 & 3.06 & 2.26 & 1.80 & 1.60 & 13.46 & 1 \\
\hline 243RZ & 5.53 & 3.40 & 2.20 & 1.73 & 1.66 & 14.53 & 1 \\
\hline 251RZ & 5.26 & 3.40 & 2.33 & 1.80 & 1.73 & 14.53 & 1 \\
\hline 263RZ & 4.46 & 3.06 & 2.26 & 2.00 & 2.06 & 13.86 & 1 \\
\hline 265RZ & 5.26 & 3.46 & 2.73 & 2.13 & 1.93 & 15.60 & 1 \\
\hline 266RZ & 5.46 & 3.73 & 2.73 & 1.73 & 1.60 & 15.26 & 1 \\
\hline 269RZ & 5.13 & 3.26 & 2.66 & 2.06 & 1.93 & 15.06 & 1 \\
\hline 270RZ & 5.26 & 3.40 & 2.33 & 1.73 & 1.73 & 14.46 & 1 \\
\hline 271RZ & 4.73 & 3.00 & 2.40 & 1.66 & 1.66 & 13.46 & 1 \\
\hline 272RZ & 5.06 & 3.26 & 2.66 & 2.06 & 1.86 & 14.93 & 1 \\
\hline 273RZ & 5.26 & 3.40 & 2.33 & 1.93 & 1.93 & 14.86 & 1 \\
\hline 274RZ & 7.93 & 8.26 & 7.80 & 7.66 & 7.90 & 39.56 & 2 \\
\hline 275RZ & 6.60 & 5.60 & 4.93 & 5.43 & 5.33 & 27.90 & 1.33 \\
\hline 276RZ & 7 & 7.46 & 7.86 & 7.23 & 7.56 & 37.13 & 1.53 \\
\hline $26 \mathrm{KS}$ & 5.2 & 3.80 & 2.80 & 1.73 & 1.60 & 15.13 & 1 \\
\hline $28 \mathrm{KS}$ & 4.46 & 3.06 & 2.33 & 1.66 & 1.53 & 13.06 & 1 \\
\hline $30 \mathrm{KS}$ & 4.66 & 2.93 & 2.46 & 2.20 & 2.00 & 14.26 & 1 \\
\hline $33 \mathrm{KS}$ & 5.33 & 3.46 & 2.73 & 1.86 & 1.86 & 15.26 & 1 \\
\hline $34 \mathrm{KS}$ & 4.93 & 3.40 & 2.80 & 1.93 & 1.80 & 14.86 & 1 \\
\hline $35 \mathrm{KS}$ & 5.2 & 3.60 & 2.53 & 2.00 & 1.93 & 15.26 & 1 \\
\hline $38 \mathrm{KS}$ & 5.53 & 3.13 & 2.33 & 1.80 & 1.60 & 14.40 & 1 \\
\hline $39 \mathrm{KS}$ & 5.06 & 3.33 & 2.73 & 2.13 & 1.80 & 15.06 & 1 \\
\hline $40 \mathrm{KS}$ & 4.46 & 2.66 & 2.06 & 1.66 & 1.53 & 12.40 & 1 \\
\hline $43 \mathrm{KS}$ & 5.06 & 3.00 & 2.26 & 1.93 & 2.00 & 14.26 & 1 \\
\hline $56 \mathrm{KS}$ & 5.13 & 3.26 & 2.20 & 1.46 & 1.53 & 13.60 & 1 \\
\hline $57 \mathrm{KS}$ & 5.2 & 3.06 & 2.46 & 1.93 & 1.80 & 14.46 & 1 \\
\hline $61 \mathrm{KS}$ & 6.26 & 4.73 & 4.46 & 5.26 & 5.40 & 26.13 & 1.06 \\
\hline $62 \mathrm{KS}$ & 7.13 & 5.13 & 5.93 & 6.40 & 5.93 & 30.53 & 1.20 \\
\hline $63 \mathrm{KS}$ & 5.06 & 3.20 & 2.26 & 1.26 & 1.46 & 13.26 & 1 \\
\hline $65 \mathrm{KS}$ & 5 & 3.00 & 2.53 & 1.66 & 1.66 & 13.86 & 1 \\
\hline $66 \mathrm{KS}$ & 5.53 & 3.46 & 2.33 & 1.80 & 1.66 & 14.80 & 1 \\
\hline $67 \mathrm{KS}$ & 5.46 & 3.26 & 2.20 & 1.73 & 1.66 & 14.33 & 1 \\
\hline $72 \mathrm{KS}$ & 5 & 3.66 & 2.46 & 1.86 & 1.86 & 14.86 & 1 \\
\hline Mean & 5.67 & 3.28 & 2.84 & 3.03 & 2.94 & 17.77 & 1.01 \\
\hline $\mathrm{LSD}_{0.01}$ & 0.81 & 0.81 & 0.68 & 0.69 & 0.63 & 2.15 & - \\
\hline
\end{tabular}


Table 7. Sensorial evaluation means of fermented milk by Lactococci isolates

\begin{tabular}{cccccccc} 
Isolates & \multicolumn{7}{c}{ Mean } \\
\cline { 2 - 9 } & Color (10) & Taste (10) & Smell (10) & $\begin{array}{c}\text { Texture(10 } \\
\text { ) }\end{array}$ & $\begin{array}{c}\text { Appearance(10 } \\
\text { ) }\end{array}$ & Total (50) & Over all \\
\hline $37 \mathrm{RZ}$ & 6.73 & 4.86 & 5.2 & 5.73 & 5.8 & 28.33 & 1 \\
\hline $66 \mathrm{RZ}$ & 7.4 & 5.13 & 5.46 & 5.13 & 5 & 28.13 & 1 \\
\hline $72 \mathrm{RZ}$ & 5.5 & 1.13 & 1.80 & 5.80 & 5.33 & 19.6 & 1 \\
\hline $124 \mathrm{RZ}$ & 8.7 & 8.6 & 8.53 & 8.13 & 8.4 & 42.4 & 2 \\
\hline $23 \mathrm{KS}$ & 7.46 & 5.33 & 4.86 & 4.33 & 3.93 & 25.93 & 1 \\
\hline Mean & 7.17 & 5.01 & 5.17 & 5.82 & 5.69 & 28.88 & 1.2 \\
\hline $\mathrm{LSD}_{0.01}$ & 0.50 & 0.65 & 0.60 & 0.72 & 0.65 & 2.01 & - \\
\hline
\end{tabular}

Overall 1: non acceptable Overall 2: acceptable

Overall; $2>$ overall $>1$; refer to different overall acceptability between panelists

Table 8. Sensorial evaluation means of fermented milk by Enterococci isolates

\begin{tabular}{|c|c|c|c|c|c|c|c|}
\hline \multirow{2}{*}{ Isolates } & \multicolumn{7}{|c|}{ Mean } \\
\hline & Color (10) & Taste(10) & Smell (10) & Texture(10) & Appearance(10) & Total (50) & Over all \\
\hline $18 \mathrm{RZ}$ & 5.86 & 1.73 & 1.00 & 1.73 & 1.93 & 12.26 & 1 \\
\hline 19RZ & 6.86 & 1.66 & 0.93 & 3.13 & 2.26 & 14.86 & 1 \\
\hline $26 \mathrm{RZ}$ & 6.73 & 1.53 & 0.73 & 4.26 & 4.06 & 17.33 & 1 \\
\hline $29 \mathrm{RZ}$ & 6.40 & 1.26 & 0.93 & 6.33 & 6.33 & 21.26 & 1 \\
\hline 30RZ & 7.00 & 1.46 & 0.93 & 6.06 & 6.20 & 21.66 & 1 \\
\hline $33 \mathrm{RZ}$ & 7.26 & 1.26 & 0.86 & 5.93 & 5.40 & 20.73 & 1 \\
\hline $35 \mathrm{RZ}$ & 6.86 & 1.33 & 0.80 & 6.46 & 6.00 & 21.46 & 1 \\
\hline $36 \mathrm{RZ}$ & 7.06 & 1.26 & 0.86 & 6.66 & 6.60 & 22.46 & 1 \\
\hline $38 \mathrm{RZ}$ & 6.60 & 1.66 & 1.66 & 5.26 & 5.13 & 20.33 & 1 \\
\hline $43 \mathrm{RZ}$ & 7.00 & 2.60 & 2.46 & 6.40 & 6.46 & 24.93 & 1 \\
\hline $44 \mathrm{RZ}$ & 6.66 & 1.53 & 1.86 & 4.80 & 4.53 & 19.40 & 1 \\
\hline $45 \mathrm{RZ}$ & 6.66 & 1.53 & 1.13 & 6.13 & 5.86 & 21.33 & 1 \\
\hline 49RZ & 6.53 & 1.20 & 1.00 & 5.60 & 5.06 & 19.40 & 1 \\
\hline 50RZ & 6.80 & 2.20 & 1.13 & 6.33 & 6.33 & 22.80 & 1 \\
\hline 51RZ & 7.86 & 2.80 & 1.66 & 7.06 & 7.26 & 26.66 & 1 \\
\hline 58RZ & 7.20 & 2.40 & 1.06 & 6.86 & 6.33 & 23.86 & 1 \\
\hline 60RZ & 7.06 & 1.40 & 1.13 & 6.86 & 6.46 & 22.93 & 1 \\
\hline 64RZ & 6.86 & 1.93 & 1.26 & 3.00 & 3.80 & 16.86 & 1 \\
\hline 73RZ & 5.53 & 1.46 & 1.00 & 5.53 & 5.00 & 18.53 & 1 \\
\hline 74RZ & 6.80 & 2.26 & 3.80 & 4.46 & 4.66 & 22.00 & 1 \\
\hline $82 \mathrm{RZ}$ & 6.86 & 1.73 & 0.93 & 4.20 & 4.06 & 17.80 & 1 \\
\hline $83 \mathrm{RZ}$ & 6.86 & 1.13 & 0.73 & 3.93 & 3.93 & 16.60 & 1 \\
\hline 89RZ & 6.93 & 2.46 & 1.86 & 4.93 & 5.13 & 21.33 & 1 \\
\hline 90RZ & 7.26 & 2.73 & 1.80 & 5.53 & 5.20 & 22.53 & 1 \\
\hline 98RZ & 6.06 & 1.20 & 0.80 & 4.33 & 4.13 & 16.53 & 1 \\
\hline 99RZ & 7.20 & 2.80 & 3.33 & 6.13 & 6.20 & 25.66 & 1 \\
\hline 100RZ & 6.80 & 3.40 & 3.93 & 6.46 & 5.80 & 26.40 & 1 \\
\hline 119RZ & 4.86 & 1.73 & 1.00 & 0.93 & 1.06 & 9.60 & 1 \\
\hline 136RZ & 5.26 & 3.53 & 3.66 & 4.40 & 4.06 & 20.93 & 1 \\
\hline 137RZ & 5.53 & 2.20 & 2.33 & 5.06 & 4.93 & 20.06 & 1 \\
\hline 141RZ & 6.86 & 3.53 & 2.80 & 6.20 & 6.26 & 25.66 & 1 \\
\hline 142RZ & 7.00 & 3.73 & 3.13 & 5.86 & 5.13 & 24.86 & 1 \\
\hline 148RZ & 6.73 & 3.86 & 3.20 & 6.33 & 5.80 & 25.93 & 1 \\
\hline
\end{tabular}




\begin{tabular}{|c|c|c|c|c|c|c|c|}
\hline 150RZ & 6.73 & 3.20 & 2.73 & 1.93 & 1.73 & 16.33 & 1 \\
\hline
\end{tabular}

Con. Table 8.

\begin{tabular}{|c|c|c|c|c|c|c|c|}
\hline \multirow[b]{2}{*}{ Isolates } & \multicolumn{7}{|c|}{ Mean } \\
\hline & $\begin{array}{l}\text { Color } \\
(\mathbf{1 0})\end{array}$ & $\begin{array}{l}\text { Taste } \\
\text { (10) }\end{array}$ & $\begin{array}{c}\text { Smell } \\
(10)\end{array}$ & $\begin{array}{c}\text { Texture } \\
(10)\end{array}$ & $\begin{array}{c}\text { Appearance } \\
\text { (10) }\end{array}$ & $\begin{array}{l}\text { Total } \\
\text { (50) }\end{array}$ & Over all \\
\hline $155 \mathrm{RZ}$ & 6.73 & 3.66 & 2.53 & 6.06 & 6.40 & 25.40 & 1 \\
\hline $167 \mathrm{RZ}$ & 6.73 & 3.66 & 1.66 & 6.66 & 5.93 & 23.66 & 1 \\
\hline 168RZ & 7.00 & 0.86 & 0.53 & 1.00 & 0.80 & 10.20 & 1 \\
\hline 219RZ & 6.73 & 2.80 & 2.60 & 3.66 & 3.46 & 19.26 & 1 \\
\hline 220RZ & 6.80 & 3.06 & 2.13 & 6.26 & 5.53 & 23.80 & 1 \\
\hline $227 \mathrm{RZ}$ & 6.73 & 3.26 & 1.73 & 5.13 & 5.00 & 21.86 & 1 \\
\hline 228RZ & 6.73 & 3.06 & 2.60 & 5.26 & 4.86 & 22.53 & 1 \\
\hline $233 \mathrm{RZ}$ & 6.73 & 3.26 & 2.00 & 6.00 & 5.26 & 23.26 & 1 \\
\hline $254 \mathrm{RZ}$ & 6.13 & 2.60 & 2.86 & 2.66 & 3.20 & 17.46 & 1 \\
\hline $255 \mathrm{RZ}$ & 7.00 & 1.80 & 1.66 & 3.46 & 3.13 & 17.06 & 1 \\
\hline $256 \mathrm{RZ}$ & 6.06 & 1.60 & 1.06 & 3.33 & 3.46 & 15.53 & 1 \\
\hline 260RZ & 6.00 & 2.06 & 2.13 & 3.06 & 3.06 & 16.33 & 1 \\
\hline 261RZ & 5.40 & 1.93 & 1.26 & 3.20 & 3.06 & 14.86 & 1 \\
\hline $262 \mathrm{RZ}$ & 5.40 & 2.00 & 1.26 & 1.93 & 1.86 & 12.46 & 1 \\
\hline $267 \mathrm{RZ}$ & 6.53 & 2.13 & 1.86 & 4.80 & 4.26 & 19.60 & 1 \\
\hline 268RZ & 7.06 & 1.66 & 1.13 & 2.33 & 2.40 & 14.60 & 1 \\
\hline $277 \mathrm{RZ}$ & 7.86 & 1.80 & 1.13 & 6.73 & 6.60 & 24.13 & 1 \\
\hline $278 \mathrm{RZ}$ & 7.53 & 1.60 & 1.13 & 6.06 & 5.80 & 22.13 & 1 \\
\hline 279RZ & 7.13 & 1.60 & 1.06 & 3.06 & 2.73 & 15.60 & 1 \\
\hline $283 \mathrm{RZ}$ & 7.40 & 2.00 & 0.73 & 4.20 & 4.00 & 18.33 & 1 \\
\hline $24 \mathrm{KS}$ & 7.33 & 1.66 & 1.06 & 1.73 & 1.60 & 13.40 & 1 \\
\hline $25 \mathrm{KS}$ & 7.46 & 1.13 & 0.86 & 1.93 & 1.00 & 12.40 & 1 \\
\hline $36 \mathrm{KS}$ & 7.26 & 2.13 & 1.26 & 1.86 & 0.93 & 13.46 & 1 \\
\hline $37 \mathrm{KS}$ & 7.40 & 1.73 & 1.13 & 3.13 & 3.06 & 16.64 & 1 \\
\hline $41 \mathrm{KS}$ & 6.00 & 1.93 & 1.60 & 6.93 & 5.53 & 22.00 & 1 \\
\hline $42 \mathrm{KS}$ & 6.53 & 2.80 & 1.66 & 6.93 & 5.53 & 23.46 & 1 \\
\hline $44 \mathrm{KS}$ & 7.20 & 1.80 & 1.00 & 2.73 & 2.53 & 15.26 & 1 \\
\hline $46 \mathrm{KS}$ & 6.26 & 1.73 & 1.00 & 2.46 & 2.80 & 14.26 & 1 \\
\hline $47 \mathrm{KS}$ & 6.86 & 1.80 & 1.26 & 5.73 & 5.26 & 20.93 & 1 \\
\hline $48 \mathrm{KS}$ & 7.13 & 1.86 & 1.13 & 5.86 & 5.33 & 21.33 & 1 \\
\hline $49 \mathrm{KS}$ & 5.93 & 1.66 & 1.06 & 3.40 & 3.80 & 15.86 & 1 \\
\hline $50 \mathrm{KS}$ & 6.73 & 1.66 & 1.06 & 4.13 & 4.00 & 17.60 & 1 \\
\hline $51 \mathrm{KS}$ & 6.53 & 1.80 & 0.80 & 6.13 & 5.40 & 20.66 & 1 \\
\hline $52 \mathrm{KS}$ & 6.93 & 1.86 & 0.73 & 3.46 & 4.06 & 17.06 & 1 \\
\hline $54 \mathrm{KS}$ & 7.40 & 1.73 & 1.00 & 3.20 & 2.73 & 16.06 & 1 \\
\hline $55 \mathrm{KS}$ & 6.80 & 1.73 & 1.00 & 4.20 & 4.06 & 17.80 & 1 \\
\hline $58 \mathrm{KS}$ & 7.53 & 1.73 & 1.00 & 4.00 & 3.80 & 18.06 & 1 \\
\hline $59 \mathrm{KS}$ & 7.40 & 2.06 & 1.20 & 6.13 & 6.13 & 22.93 & 1 \\
\hline $60 \mathrm{KS}$ & 7.33 & 1.93 & 1.40 & 4.06 & 3.26 & 18.00 & 1 \\
\hline $64 \mathrm{KS}$ & 7.40 & 1.60 & 0.66 & 2.46 & 2.86 & 15.00 & 1 \\
\hline $68 \mathrm{KS}$ & 7.13 & 1.53 & 0.66 & 4.40 & 4.20 & 17.93 & 1 \\
\hline $69 \mathrm{KS}$ & 5.26 & 1.33 & 0.93 & 4.66 & 4.40 & 16.60 & 1 \\
\hline $71 \mathrm{KS}$ & 5.86 & 1.53 & 0.86 & 5.80 & 5.20 & 19.26 & 1 \\
\hline
\end{tabular}




\begin{tabular}{lrllllll}
\hline Mean & 6.75 & 2.15 & 1.60 & 4.71 & 4.46 & 19.70 & 1.01 \\
\hline LSD $_{0.01}$ & 0.57 & 0.58 & 0.55 & 0.76 & 0.68 & 1.87 & - \\
\hline \multicolumn{7}{c}{ Overall 1: non acceptable } & \multicolumn{7}{c}{ Overall 2: acceptable }
\end{tabular}

Group C isolates; the smell and the taste were not very pleasant, the curd was very bitter, this was found with isolates (16RZ, 55RZ and 266RZ). Haddadin, 2005 found similar result with some tested strains of lactobacilli. Other G.C isolates in this study, produced sweet taste and strong odor in fermented milk. There are significant differences between panelists to accept this taste Tables (3 and 6). The isolate (274 RZ) was acceptable by all panelists. Its curd was soft, smooth, and homogenous; and the sweet taste was accepted.

Lactococci isolates confer to the curd certain pleasant odor and compact texture except isolate (72RZ). The (124RZ) isolate was received the highest overall acceptability level when compared to all other lactococci. The obtained results in Table (7) clearly showed that there are significant differences $(\mathrm{P} \leq 0.01)$ in sensorial evaluation means for lactococci isolates.

Enterococci isolates, the curd was very unpleasant taste and cowshed odder. Using the isolate (246RZ); the curd presents marked defect with a strong rancidity, on the other hand, (238RZ) was the best isolates in flavor acceptability without defects. Data in Table(8) indicate the differences between the sensorial evaluation means of enterococci isolates.

\section{CONCLUSION}

The domination of obligatory heterofermentative and Enterococcus spp. among the associated LAB was established during fermentation process of Egyptian Kishk, while the facultative heterofermentative isolates were predominant. These were significant differences in organoleptic properties among isolates in fermented milk. Hemolytic activity of most isolates were $\gamma$ hemolytic. To obtain cereal fermented milk with flavor characteristics similar to those of artisanal product, the isolated lactic acid bacteria should first be identified completely and tested in mixed cultures based on their technological properties. In this study, 15 isolates, which had a good organoleptic characteristics and no hemolytic activity, were selected for complied identification. Based on these results, future works will be done to selecte a new starter culture to produce fermented milk like Laban Zeer for its using in Kishk manufacturing.

\section{REFERENCES}

Abd El-Malek, Y. and Demerdash, M. (1970). Proceedings of the 2 nd Conference of Microbiology, Kishk. p153. Cairo.

Abd El Rahman,I.E.; DirarH.A. and Osman,M.A. (2009). Microbiological and biochemical changes and sensory evaluation of camel milk fermented by selected bacterial starter cultures. African Journal of Food Science Vol. 3. (12) pp. 398-405.

Abd El-Salam, M.H. (2011). Fermented Milks, Middle Eastern Fermented Milks . Encyclopedia of Dairy Sciences (Second Edition), 2011, pp. 503-506.

Abou-Donia, S.A. (1984). Egyptian fresh fermented milk products. N. Z. J. Dairy Sci. Technol., 19 : pp. 7-18.

Ahmed, Z.S. and Hassan-Wassef, H. (2010). Literature review and Back- ground information of Kishk Sa'eedi. African food tradition revisited by research .After Project.

Atia, I. A. and Khattab, A. A. (1985). Microbiological and chemical studies on Kishk. Alex.Sci.Exch., 6: pp. 63-71.

Axelsson, L. (1993). Lactic acid bacteria: classification and physiology. In: Salminen, S.J.,Wright, A. (Eds.), Lactic Acid Bacteria. Marcel Dekker, Inc., New York, pp. 1-6.

Buffa, M.; Morais, J., Jiménez-Belenguer, A., HernundezGiménez, E. and Guamis, B. (2005). Technological characterization of lactic acid bacteria isolated from raw ewes' milk for cheese making. Milchwissenschaft, 61: pp. $404-407$.

Damir, A. A.; Salama, A.A. and Safwat Mohamed,M. (1992). Acidity, microbial, organic and free amino acids development during fermentation of skimmed milk, Kishk. Food Chemistry 43: pp.265-269.

Demerdash, M.A. (1960).Studies in microbiology of fermented milk common in Egypt. M.Sc. Thesis, Faculty of Agriculture, Cairo University pp. 92 .

El-Sadek, G.M.; Zawahry, M.R.; Mahmoud ,S.A.Z. and Abd El Motteleb, L.(1958). Chemical composition of Egyptain Kishk. Indian J.Dairy Sci.,11: pp.6

El Soda, M.; Ahmed, N.; Omran, N.; Osman, G. and Morsi, A. (2003). Isolation, identification and selection of lactic acid bacteria cultures for cheese making. J. Agric. Sci., 15: pp.51.

Franz, C. M. A. P ; Holzapfel, W. H. and Stiles, M. E. (1999). Enterococci at the crossroads of food safety?" International Journal of Food Microbiology, Vol. 47, No. 1-2, pp. 1-24.

Haddadin, J.S.Y. (2005). Kinetic studies and sensorial analysis of Lactic Acid Bacteria isolated from white 
cheese made from sheep raw milk. Pakistan Journal of Nutrition, 4, (2): pp.78-84.

Hamad, A. M. and Fields, M. L. (1982). Preliminary evaluation of a new type of Kishk made from whey. $J$. Food Sci., 47: pp.1140

Jett, B. D.; Huycke, M. M. and Gilmore, M. S. (1994). Virulence of Enterococci," Clinical Microbiology Reviews, Vol. 7, No. 4, pp. 462-478.

Kandler, O. and Weiss, N. (1986). Regular non-sporing gram positive rods, In: P. H. A. Sneath, N. Mair, M. E. Sharpeand J. G. Hoit (Eds). pp. 1208-1234. Bergey's Manual of systematic Bacteriology 2. Willims and Wilkins Baltimore.

Maragkoudakis, P.A.; Mountzouris, K. C. and Psyrras, D. (2009). Functional properties of novel protective lactic acid bacteria and application in raw chickenmeat against Listeria monocytogenes and Salmonella enteritidis," International Journal of FoodMicrobiology, Vol. 130, No. 3, pp. 219-226.

Maragkoudakis, P.A.; Zoumpopoulou, G.; Miaris, C.; Kalantzopoulos, G.; Pot,B. and Tsakalidou, E.(2006).
Lactobacillus strains isolated from dairy products. International Dairy Journal, 16: pp.189-199.

Shaker, R. R.; Shehata, A. M. E.; EI-Banna, A. A. and Mesallam, A. S. (1980). Microbiological and chemical changes during fermentation of turnip-wheat Kishk and tarkhina. Proc. IV Conf. Microbiol., Cairo, 24-28 Dec. 1980, Vol. 1, Soil, Food and Industrial MicrobioL, pp. 381-92.

Sharpe, M. E. (1979). Identification of lactic acid bacteria, In: F. A. Skinner and D. W. Lovelock (Eds). Identification methods for microbiologists. London: Academic Press. pp.233-259.

Steel, J.D. and Torrie, J.H. (1984). Principles and procedures of statistics. A biometrical approach. McGraw Hil Co. Inc., New York 1. 4th printing.

Tamime, A. Y. and O'Connor, T. P. (1995). Kishk: A dried fermented milk/cereal mixture. International Dairy Journal, 5:pp. 109-128.

\section{المالغص المري \\ النعرف المبئي لبكتربا حصض اللكتيك المعزوله خلال تخمر الهثك المصري

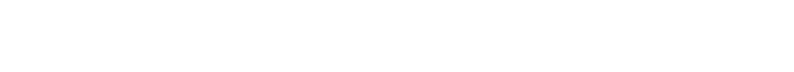

خلل صناعة الكثك هـ البكتريا العصوية لالكتوبلسيالس

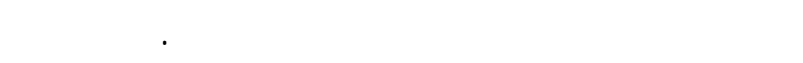

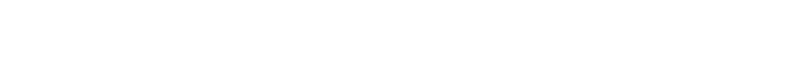

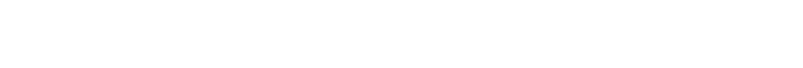

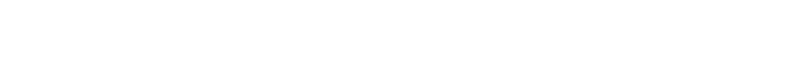

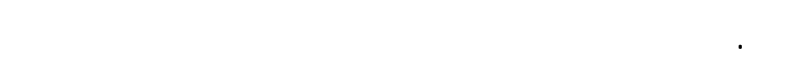

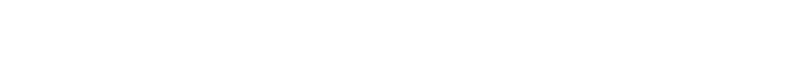

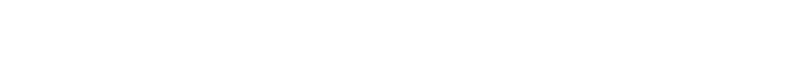
خلا صناعه المثك جميع العزلات غير محلله للمه (من

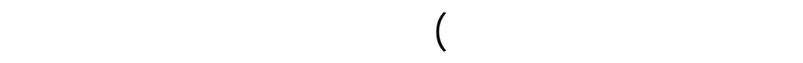

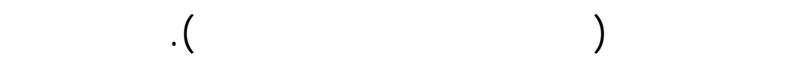

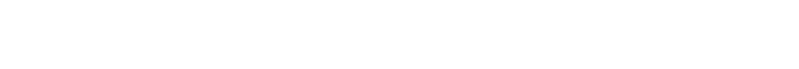

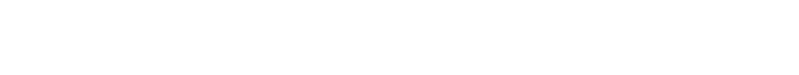
الهسيه(اللون- الطعم - الرائحه- الفظهر - القبل العلم) بين
الهوف من لجراء الدرلسة هولختبار درلسة تاثير المنان والبيتا يعتبر الكثك المصري مصدر رئيسي في الوجبلت الفراء

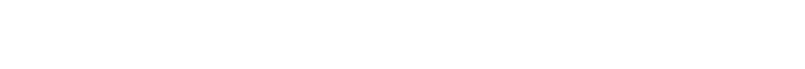

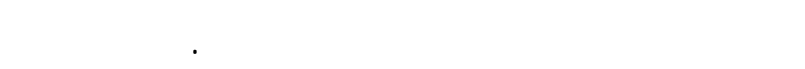

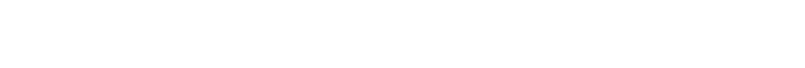

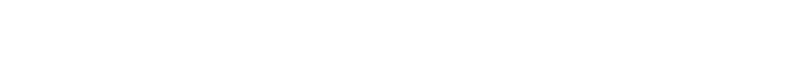

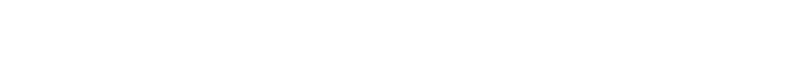
بكتريا حمض اللكتيك المسئولة كن التخمرات بصورة

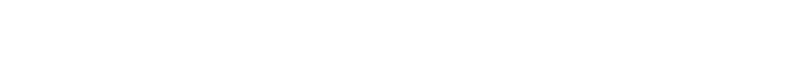

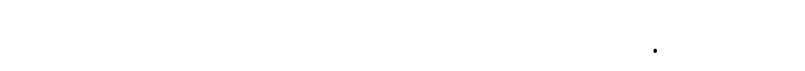

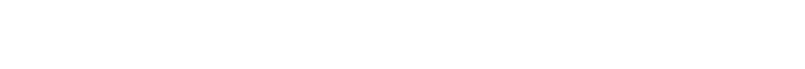
المبئي عليها حتي يمكن التتخلب بعض البكن السلالات الني تخدمر

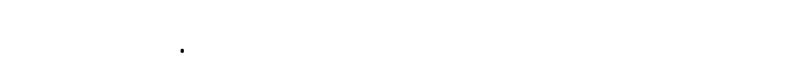
النتائج المتحصل عليها لن بكتيريا حمض اللكتيك اللنيائئة 
العزلات عند مستوى معنوية ا ·, · ولذك مُ انتخلب خمسة عثرسلالة والتي كانت الافضل فى الخواص الحسيه وذلك لتكوين بادى التهسين صناعه الكثك المصري. 